\title{
Notas sobre Apterocaulus (Coleoptera, Cerambycidae, Prioninae)
}

\author{
Osvaldo R. Di Iorio ${ }^{1} \&$ Antonio Santos-Silva²
}

1. Soler 1167, Caixa Postal 1615, Grand Bourg, Buenos Aires, Argentina.

2. Museu de Zoologia, Universidade de São Paulo, Caixa Postal 42594, 04299-970 São Paulo, SP, Brasil.

\begin{abstract}
Notes on Apterocaulus (Coleoptera, Cerambycidae, Prioninae). The taxonomic situation of Apterocaulus Fairmaire, 1864 and the validity of Apterocaulus heterogama heterogama (Burmeister, 1861) are discussed. Apterocaulus heterogama durnfordii (Burmeister, 1879) is considered as valid subspecies, being accepted Di Iorio's (2002) arguments.
\end{abstract}

KEYWORDS. Cerambycidae, Prionini, synonymy, taxonomy.

RESUMO. A situação taxonômica de Apterocaulus Fairmaire, 1864 e a validade de Apterocaulus heterogama heterogama (Burmeister, 1861) são discutidas. Apterocaulus heterogama durnfordii (Burmeister, 1879) é considerada como subespécie válida, sendo aceito os argumentos de Di Iorio (2002).

PAlaVRAS-CHAVE. Cerambycidae, Prionini, sinonímia, taxonomia.

Algumas espécies de Cerambycidae apresentam um histórico taxonômico notável. Entre elas, sem dúvida, Apterocaulus heterogama (Burmeister, 1861) destaca-se tanto por seu status (espécie válida, sinônimo, nomen nudum), quanto por sua alocação genérica (Psalidognathus Gray, 1831, Micropsalis Burmeister, 1861 ou Apterocaulus Fairmaire, 1864).

Recentemente, SAntos-Silva (2003) restabeleceu Apterocaulus Fairmaire, 1864 (Cerambycidae, Prioninae, Prionini) para substituir Micropsalis Burmeister, 1861, homônimo de Micropsalis Meyer, 1859 (Crustacea), apresentou uma retrospectiva dos gêneros Micropsalis Burmeister e Apterocaulus, considerou A. germainii Fairmaire, 1864 sinônimo de A. heterogama (Burmeister, 1861) e não emitiu parecer sobre a validade de $A$. heterogama durnfordii (Burmeister, 1879). Essa subespécie havia sido colocada na sinonímia de $A$. heterogama (=Micropsalis heterogama Burmeister, 1861) por MONnÉ \& GIESBERT (1994). Di IORIO (1996) referiu-se à subespécie como Psalidognathus germaini durnfordi [trabalho no prelo, quando o catálogo de MonNé \& GIESBERT (1994) foi publicado].

Di Iorio (2002) defendeu a validade do gênero Micropsalis Burmeister, mas não considerou o epíteto heterogama como válido. Os artigos de Di IORIO (2002) e SANTOS-SILVA (2003) foram publicados com poucos meses de diferença entre si e nenhum dos dois autores conhecia a existência do trabalho do outro.

Com relação a Micropsalis Burmeister, a discussão encerra-se com sua condição de homônimo, mas julgamos necessário esclarecer algumas questões tanto sobre o gênero, quanto sobre Micropsalis heterogama (=A. heterogama).

Retrospectiva das principais publicações, envolvendo transferências, sinonímias, revalidações e homonímia:

1861 - BuRMEISTER descreveu o gênero Micropsalis com a espécie $M$. heterogama;
1864 - FAIRMAIRE estabeleceu o gênero Apterocaulus com duas espécies: A. germainii e A. marginipennis;

1865 - BURMEISTER redescreveu Micropsalis heterogama;

1869 - LACORDAIRE estabeleceu a sinonímia de Apterocaulus e Micropsalis Burmeister, salientando tratar-se de um nome posterior há três anos daquele publicado por Burmeister. Também sinonimizou: $A$. germainii $=M$. heterogama $;$ A. marginipennis $=$ Micropsalis servillei (Blanchard, 1851);

1877 - THOMSON estabeleceu o gênero Acalodegma para M. servillei;

1879 - BURMEISTER descreveu Micropsalis durnfordii;

1910 - LAMEERE considerou Micropsalis como subgênero de Psalidognathus Gray, 1831 e M. durnfordi como sinônimo de $M$. heterogama;

1912 - LAMEERE considerou o ano de 1865 como a data válida da descrição de $M$. heterogama. Assim, sinonimizou Micropsalis Burmeister, 1865 com Apterocaulus e confirmou a sinonimização de $M$. heterogama (sinônimo júnior) com $A$. germaini (sinônimo sênior) e manteve Apterocaulus como subgênero de Psalidognathus;

1913 - LAMEERE, no Coleopterorum Catalogus (vol. 52), manteve Psalidognathus (Apterocaulus) germaini. Micropsalis durnfordi foi considerada uma variedade de $P$. (A.) germaini;

1919 - LAMEERE, no Genera Insectorum (vol. 172), manteve a mesma colocação do Coleopterorum Catalogus;

1930 - Gemignani utilizou o nome Apterocaulus durnfordii em seu trabalho. Desconhecemos a razão pela qual esse autor considerou Apterocaulus como gênero e não como subgênero (provavelmente, apenas um erro);

1946 - BlackWelder publicou seu Checklist com a mesma colocação do Coleopterorum Catalogus;

1953 - BosQ apresentou uma retrospectiva de Apterocaulus, mantendo-o como subgênero de Psalidognathus e considerou $M$. durnfordii como subespécie de $P$. (A.) germaini (sic); 
1994 - Monné \& Giesbert revalidaram Micropsalis Burmeister e arrolaram: Apterocaulus = Micropsalis Burmeister; A. germainei $($ sic $)=M$. heterogama. $M$. durnfordii foi considerada um sinônimo de $M$. heterogama.

1995 - Monné manteve o que foi estabelecido por Monné \& GIESBERT (1994);

1996 - Di IoRIo publicou trabalho sobre Psalidognathus germaini durnfordi, com dados sobre as primeiras plantas hospedeiras conhecidas (e outras erroneamente atribuídas), biologia das larvas e distribuição geográfica (incluindo, por erro, a localidade-tipo de M. heterogama, isto é, Mendoza, Argentina);

2002 - Di IORIO também considerou Micropsalis Burmeister, 1861 como gênero válido, $M$. heterogama Burmeister, 1861 nomen nudum e a subespécie $M$. germaini durnfordi como válida. Assim: Apterocaulus = Micropsalis; M. heterogama = M. germaini germaini; M. durnfordi $=$ M. germaini durnfordi;

2002 - Elgueta \& Cerda publicaram nova espécie de Prioninae (Acalodegma vidali) e teceram comentários sobre Micropsalis, M. heterogama e M. durnfordii;

2003 - SANTOS-Silva, desconhecendo o trabalho de Di IORIO (2002), restabeleceu Apterocaulus para substituir Micropsalis Burmeister devido à homonímia, considerou A. heterogama (Burmeister, 1861) como espécie válida e não emitiu opinião sobre M. durnfordii Burmeister, 1879 nem sobre a posição das subespécies, justificando: "No specimens of $A$. heterogama durnfordii were examined and the considerations about this subspecies name could not be evaluated".

\section{Apterocaulus Fairmaire, 1864}

Micropsalis BURMEISTER, 1861:314; 1865:157; LACORDAIRE, 1869:43; BurmeIsTER, 1879:196; GERMAIN, 1897:426 (comentário); LAMEERE, 1913:66 (cat.; in syn.); 1919:121 (in syn.); BLACKWELDER, 1946:555 (cat.; in syn.); BosQ, 1953:70 (in syn.); Monné \& GIEsBert, 1994:14 (cat.; reval.); MonnÉ, 1995:61 (cat.); Di Iorio, 2002: 3; Elgueta \& Cerda, 2002:12, 20; Monné, 2002:22; Bachmann \& Di Iorio, 2002:70 (síntipo); Santos-Silva, 2003:479 (hom., nom. preoc. por Micropsalis Meyer, 1859 - Crustacea).

Apterocaulus FAIRMAIRE, 1864:267; THOMSON, 1864:496; LACORDAIRE, 1869:43 (syn.); BuRMEISTER, 1879:196 (in syn.); GERMAIN, 1897:426 (comentário); LAMEere, 1910:378 (in syn.); BRuch, 1912:183 (cat.); Monné \& Giesbert, 1994:14 (cat.; syn.); Monné, 1995:61 (cat.; in syn.); Di Iorio, 2002:3 (in syn.); Santos-Silva, 2003:479 (reval.).

Psalidoganathus (Micropsalis); LAMEERE, 1910:378; 1912:175 (syn.).

Psalidognathus (Apterocaulus); LAMEERE, 1912:175 (reval.; status subgen.); 1913:66 (cat.); BRUCH, 1915:552 (cat.); LAMEERE, 1919:121; Porter, 1933:207; BLACKWELdER, 1946:555 (cat.); MonNÉ, 1995:61 (cat.; in syn.).

\section{Apterocaulus heterogama heterogama (Burmeister, 1861)}

Micropsalis heterogama BURMEISTER, 1861:314; 1865:157; Gemminger \& Harold, 1872:2755; Burmeister, 1879:196; LAMEERE, 1912:175 (syn.); 1913:66 (cat.; in syn.); BRUCH, 1912:183 (cat.; in syn.); LAMEERE, 1919:122 (in syn.); Blackwelder, 1946:555 (cat.; in syn.); Monné \& Giesbert, 1994:14 (cat.; reval.); Monné, 1995:61 (cat.); RoJas Lanus, 1997:21 (distr.); Di Iorio, 2002:14 (nom. nud.); Monné, 2002:22 (hosp.); 2004:30, 71 (hosp.).
Apterocaulus Germainii FAIRMAIRE, 1864:268; THOMSON, 1864:496; LACORDAIRE, 1869:44 (syn.).

Psalidognahtus (Micropsalis) heterogama; LAMEERE, 1910:379; 1912:175 (syn.).

Apterocaulus Germaini; LAMEere, 1910:379 (in syn.); BRUCH, 1912:183 (cat.); REED, 1912:40, 41 (fig.13).

Psalidognathus (Apterocaulus) Germaini; LAMEERE, 1912:175 (reval.); 1913:66 (cat.); 1919:122; MElzer, 1919:131.

Apterocaulus durnfordii; GeMignani, 1930:634 (fig. 23), 636 (distr.).

Psalidoganathus germaini; BLACKWELDER, 1946:555 (cat.); CHIESAMolinARI, 1942:441; Ringuelet, 1955:99.

Psalidognathus (Apterocaulus) germaini; BRUCH, 1915:552 (cat.). Micropsalis germaini germaini; DI IORIO, 2002:14.

Apterocaulus germainei; MonNÉ \& GIESBERT, 1994:14 (cat.; syn.). Psalidognathus germaini durnfordi (non Burmeister, 1879); Di IoRIo, 1996:87 (partim).

Apterocaulus heterogama; SANTOS-SILVA, 2003:479 (reval.; comb. nov.). Apterocaulus germainii; SANTOS-Silva, 2003:479 (syn.).

\section{Apterocaulus heterogama durnfordii (Burmeister, 1879)}

Micropsalis Durnfordii Burmeister, 1879:196; LAMEere, 1883:5 (cat.); ANôNIMO, 1898:xvi; LAMEERE, 1910:379 (syn.).

Apterocaulus Dunfordi; BRUCH, 1912:183 (cat.).

Psalidognathus (Apterocaulus) germaini var. Dunfordi; BRUCH, 1915:552.

Psalidognathus (Apterocaulus) germaini var. durnfordi; RINGUELET, 1955:99.

Apterocaulus Durnfordi; Wendt, 1984:332 (síntipos); Monné, 1995:61 (cat.; in syn.); 2002:23 (in syn.).

Psalidognathus (Apterocaulus) germaini (nec Fairmaire, 1879); Porter, 1933:207; Buck, 1959:580 (distr.).

Psalidognathus (Apterocaulus) Germaini var. Durnfordi; LAMEere, 1913:66 (cat.; reval.); 1919:122.

Psalidognathus germaini var. durnfordi; BLACKWELDER, 1946:555; Avarena, 1974:122 (distr.).

Psalidognathus (Apterocaulus) germaini durnfordi; BosQ, 1953:69; Cerda, 1974:43; Monné, 1995:61 (cat.; in syn.); 2002:23 (in syn.).

Psalidognathus (Apterocaulus) germaini durnfordi; CERDA, 1986:30 (cat.).

Psalidognathus germaini durnfordi; StaneK, 1985:253; BARriga et al., 1993:84 (hosp.); Di IoRIo, 1996:87 (biol., distr., hosp.). Micropsalis durnfordii; Monné \& GiesBert, 1994:14 (syn.).

Micropsalis durnfordi; RoJas LANUs, 1997:21-22 (distr., biol. hosp.). Psalidognathus germaini (non Fairmaire, 1864); RoJas LANUS, 1997:22 fig. (fêmea).

Micropsalis germaini durnfordi; Di IoRIO, 2002:8 (reval.).

Apterocaulus heterogama durnfordii; SANTOS-SILVA, 2003:479.

Discussão taxonômica. SANTos-Silva (2003) designou Apterocaulus germainii Fairmaire, 1864 como espécie-tipo de Apterocaulus e comentou: "MonNÉ (1995) kept $M$. durnfordii under synonymy and stated about Apterocaulus: "Type species, Apterocaulus germainii Fairmaire, 1864, monotypy". However, based on data above, Fairmaire (1864) erected Apterocaulus for two species: A. germainii and A. marginipennis. This way, the type species was never designated for the genus". Di IORIO (2002) considerou o epíteto heterogama validado em BURMEISTER (1865) e mencionou como espécie-tipo de Micropsalis Burmeister: "Micropsalis heterogama Burmeister, 1861 [by monotypy; nomen nudum]". Uma espécie nominal só pode ser considerada como espécietipo se esse nome for válido, o que não é o caso de um nomen nudum (ICZN, Art. 67.2.1).

Se um gênero for monotípico, os caracteres genéricos são inteiramente compartilhados pela espécie. Dessa forma, quando Burmeister atribuiu um nome à 
espécie (M. heterogama), esta se tornou válida (ICZN, Art. 12.2.6) e, conseqüentemente, é a espécie-tipo por monotipia. Assim, Di IORIO (2002) considerou erroneamente $M$. heterogama Burmeister, 1861 como um nomen nudum, quando na realidade não era. Apesar do nome genérico ser inválido, por sua homonímia, $M$. heterogama Burmeister, 1861, não perde sua condição de espécie-tipo (ICZN, Art. 67.1.2).

Pelo exposto, observa-se que Micropsalis Burmeister é um nome inválido, por homonímia (SANTOSSilva, 2003), Apterocaulus heterogama (Burmeister, 1861) é uma espécie válida e, portanto, Apterocaulus germainii Fairmaire, 1864 deve ser considerada sinônima. Como a espécie descrita por BURMEISTER (1879), Micropsalis durnfordii, é considerada subespécie de A. heterogama, conseqüentemente deve ser referida como A. heterogama durnfordii.

Elgueta \& Cerda (2002), acertadamente, escreveram: "Em cuanto a $M$. heterogama, ya em su descripción original Burmeister (1861:314) había establecido su estrecha asociación al suelo, citando: "Ambos sexos no son alados, por lo que viven en el suelo, bajo la maleza de los arbustos y entre los palitos y taladrando las raíces; a pesar de lo breve de la descripción, ampliada con posterioridad (BURMEISTER 1865), su autor entrega los caracteres necessarios para el adecuado reconocimiento de ella, incluyendo aspectos biológicos relevantes y aquél relativo a la extraordinaria modificación del segmento terminal de palpos en el macho; este antecedente es el que justifica la acción de MonNÉ (1995) al establecer la prioridad del nombre dado por BURMEISTER (1861), sobre la nominación posterior de FAIRMAIRE (1864, Apterocaulus germaini) para la misma especie y que fuera utilizada sin cuestionamento por todos los autores posteriores". ElgUETA \& CERDA (2002) expuseram sua dúvida sobre a ocorrência de A. heterogama no Chile, o que foi aclarado por Di IORIO (2002): "PORTER (1933) illustrates the species of Prioninae of Chile, among them a male specimen of Micropsalis germaini durnfordi, but in the text he refers to Micropsalis germaini germaini. Bose (1953) mentions that specimens from Chile correspond to Micropsalis germaini durnfordi, repeated by CERDa (1974). Distribution recorded by Monné \& GIESBERT (1995), and MonNÉ \& Hovore (2002) corresponds only to Micropsalis germaini durnfordi".

Com relação aos nomes específicos germainii, durnfordii, germaini e durnfordi, devem ser mantidas as grafias das descrições originais (ICZN, Art. 31.1.3), portanto, os dois primeiros.

Burmeister (1861) descreveu A. heterogama (originalmente em Micropsalis) com base em um casal de síntipos. Di IORIo (2002) afirmou que o exemplar fêmea, utilizado por BURMEISTER (1865) para redescrever a espécie, não é um síntipo. O síntipo fêmea foi extraviado, conforme afirmou BURMEISTER (1861) e, portanto, resta apenas o síntipo macho. BACHMANN \& Di IORIO (2002) mencionaram a existência de dois síntipos, um macho e uma fêmea, na coleção do Museo Argentino de Ciencias Naturales "Bernardino Rivadavia" (MACN), e, em nota, escreveram: "on the bottom of the drawer, "Micropsalis Nobis / Apterocaulus Firm" [sic], "Heterogama Nob. / A. germainii Firm [sic] / Mendonza" [handwritten by
Burmeister in black on white paper inside a black frame]. Monné \& Giesbert (1995) and Monné (1995) considered that Micropsalis heterogama Burmeister, 1861 has precedence over the same name of Burmeister (1865). In the mention of Micropsalis heterogama, Burmeister (1861) say that the "hembra desgraciadamente se me fue por culpa de este mismo acompañante". If 1861 is considered as the real date of publication, the female Syntype is not a type".

Embora o síntipo fêmea tenha sido perdido, sua condição como tipo não é invalidada (ICZN, Art. 74.4). Julgamos desnecessário designar o síntipo macho como lectótipo, por ser o único tipo existente, além da espécie não apresentar problemas para identificação. Com relação ao exemplar fêmea, rotulado como síntipo no MACN, acrescentamos uma etiqueta, esclarecendo sua condição de não-tipo.

WendT (1984) relacionou três síntipos de Micropsalis durnfordii na coleção do "ZMHB - Museum für Naturkunde der Humboldt-Universität" e Di IORIO (2002) comentou que provavelmente esses espécimes não são tipos. Os rótulos manuscritos desses exemplares foram escaneados pelo Dr. Johannes Frisch (ZMHB) e enviados para nós. Comparamos a caligrafia com aquela de Burmeister, presente nos rótulos da coleção do MACN, concluindo que são diferentes. Assim, pode-se inferir que os exemplares na coleção do ZMHB não são tipos. Consideramos como síntipo de Micropsalis durnfordii o exemplar comentado em Di IORIO (2002).

Agradecimentos. Aos Drs. Manfred Uhlig e Johannes Frisch (ZMHB), pelas informações e escaneamento dos rótulos dos espécimes mencionados por Wendt (1984); à Dra. Maria Helena M. Galileo (Museu de Ciências Naturais, Fundação Zoobotânica do Rio Grande do Sul), pelas críticas, sugestões e revisão do texto.

\section{REFERÊNCIAS BIBLIOGRÁFICAS}

ANôNIMO. 1898. Exhibitions. April $6^{\text {th }}$, 1898. Transactions of the Entomological Society of London, 1898:16-17.

Avarena, R. O. 1974. Insectos de La Pampa (Coleópteros). Provincia de La Pampa, Consejo Provincial de Difusión, Biblioteca Pampeana. 166 p.

Bachmann, A. O. \& Di Iorio, O. R. 2002. Types and related specimens of Cerambycidae and Disteniidae (Coleoptera) from the Museo Argentino de Ciencias Naturales "Bernardino Rivadavia", Buenos Aires, Argentina. Revista del Museo Argentino de Ciencias Naturales "Bernardino Rivadavia", Nueva Serie, 4(1):55-93.

Barriga, J. E.; Curkovic, T.; Fichet, T; Henríquez, J. L. \& Macaya, J. 1993. Nuevos antecedentes de coleópteros xilofagos y plantas hospederas en Chile, con una recopilación de citas previas. Revista Chilena de Entomologia, 20:65-91.

Blackwelder, R. E. 1946. Checklist of the coleopterous insects of Mexico, Central America, the West Indies and South America. Part 4. Bulletin of the United States National Museum, 185:551-763.

BosQ, J. M. 1953. Longicórnios del Parque Nacional Lanin, zona de San Martin de los Andes y cercania (Col. Cerambycidae). Anales del Museo Nahuel Huapi, 3:69-87.

Bruch, C. 1912. Catálogo sistemático de los Coleópteros de la República Argentina. Pars VIII. Família Cerambycidae (Prionini, Cerambycini, Lamiini). Revista del Museo de La Plata, 18:179-226.

1915. Suplemento al catálogo sistemático de los Coleópteros de la República Argentina. Pars IX. Suppl. I (Addenda et corrigenda). Revista del Museo de La Plata, 19:538-575.

Buck, P. 1959. Cerambycidae in der Sammlung des Instituto 
Anchietano de Pesquisas. Pesquisas, 3:577-609.

Burmeister, H. C. 1861. Reise durch die La Plata Staaten 1857-1860. Halle, 1:1-504.

1865. Longicornia Argentina. Systematische Uebersicht der Bockkäfer der La Plata-Staaten. Stettiner Entomologische Zeitung, 26:156-181.

1879. Briefliche Mittheilungen. (3. Nachträge zu dem Aufsatz: Longicornia Argentina, Zeitung 1865, S. 156 ff). Stettiner Entomologische Zeitung, 40:194-209.

Cerda, M. G. 1974. Revisión de los Prioninae de Chile. Revista Chilena de Entomología, 8:41-46.

1986. Lista sistemática de los cerambícidos chilenos (Coleoptera, Cerambycidae). Revista Chilena de Entomologia, 14:29-39.

Chiesa-Molinari, O. 1942. Entomologia Agrícola. Identificación y control de insectos y otros animales dañinos o útiles a las plantas. San Juán, 571 p.

Di Iorio, O. R. 1996. Biology of Psalidognathus germaini durnfordi (Burmeister, 1879) (Coleoptera: Cerambycidae: Prioninae) in Patagonia, Argentina. Giornale Italiano di Entomologia, 8:87-94.

2002. Taxonomy and systematics of Cerambycidae from Argentina: the genus Micropsalis Burmeister, 1861 (Coleoptera, Cerambycidae, Prioninae). Les Cahiers Magellanes, 17:1-22.

Elgueta, M. \& Cerda, M. 2002. Acalodegma vidali, nueva especie altoandina de Prioninae (Coleoptera: Cerambycidae) de Chile. Gayana, 66(1):11-21.

FAIRMAIRE, L. 1864. Note sur quelques coléoptéres recueillis para M. $\mathrm{Ph}$. Germain a Mendonza, das les Cordiliéres. Annales de la Société Entomologique de France, (4) 4:261-274.

Gemignani, E. V. 1930. Notas sobre coleópteros, especialmente argentinos. Revista Geográfica Americana, 1:629-638.

Gemminger, M. \& Harold, E. 1872. Catalogus Coleopterorum hucusque descriptorum synonymicus et systematicus. Monachii, 9:2669-2988.

Germain, P. 1897. Apuntes entomolójicos. Los lonjicornios chilenos. Anales de la Universidad de Chile, 97:423-459.

ICZN (International Commission on Zoological Nomenclature). 1999. International Code of Zoological Nomenclature. London, $\mathrm{xxx}+306 \mathrm{p}$.

Lacordaire, J. T. 1869. Histoire Naturelle des Insectes. Genera des Coléoptères, ou exposé méthodique et critique de tous les genres proposés jusqu'ici dans cet ordre d'insectes. Paris, Roret. v. 8, 552 p.

LAMEERE, A. 1883. Liste des cérambycides, décrits postérieurement au catalogue de Munich. Annales de la Société Entomologique de Belgique, Bruxelles, 26:1-78. 1910. Révision des prionides (Dix-septième mémoire. Prionines IV). Annales de la Société Entomologique de Belgique, 54:368-383.

1912. Révision des prionides (Vingt-deuxième mémoire. -
Addenda et Corrigenda). Mémoires de la Société Entomologique de Belgique, 21:113-188.

1913. Coleopterorum Catalogus, pars 52, Cerambycidae. Prioninae. Berlin, W. Junk. 108 p.

1919. Coleoptera, Fam. Cerambycidae, subfam. Prioninae. In: Wytsman, P. ed. Genera Insectorum. v. 172, 189 p.

Melzer, J. 1919. Os longicórnios brazileiros da subfamilia "Prioninae", tomando em consideração particular as espécies do Estado de São Paulo. Revista do Museu Paulista, 11:1-207.

MonNÉ, M. A. 1995. Catalogue of the Cerambycidae (Coleoptera) of the Western Hemisphere. Part XXII. São Paulo, Sociedade Brasileira de Entomologia. 115 p.

2002. Catalogue of the Neotropical Cerambycidae (Coleoptera) with known host plant - Part V: Subfamilies Prioninae, Parandrinae, Oxypeltinae, Anoplodermatinae, aseminae and Lepturinae. Publicações avulsas do Museu Nacional, 96:1-72.

2004. Catalogue of the Neotropical Cerambycidae (Coleoptera) with known host Plant - Part VI: host plant index. Publicações avulsas do Museu Nacional, 100:1-96.

Monné, M. A. \& Giesbert, E. F. 1994. Checklist of the Cerambycidae and Disteniidae (Coleoptera) of the Western Hemisphere. Burbank, Wolfsgarden. 410 p.

Porter, C. E. 1933. Los Prioninae de Chile. Revista Chilena de Historia Natural, 37:200-209.

ReED, C. S. 1912. Entomología económica argentina. Noticias biológicas y económicas referentes a diversos insectos dañinos a la agricultura Argentina. Mendoza, $123 \mathrm{p}$.

Ringuelet, R. 1955. Vinculaciones faunísticas de la zona boscosa del Nahuel Huapi y el domínio zoogeográfico Austral cordillerano. Notas del Museo de La Plata, Zoología, 18(160):81-121.

Rojas Lanus, D. 1997. Micropsalis heterogama Burmeister (Psalidognathus germaini y $P$. durnfordi). Revista informativa de la Asociación Amigos Del Museo "Mariposas Del Mundo", 3:21-22.

Santos-Silva, A. 2003. On two generic homonymies in Cerambycidae (Coleoptera). Revista Brasileira de Zoologia, 20(3):479-480.

Stanek, V. J. 1985. Encyclopédie des insectes Coléoptères. Paris, Gründ. 352 p.

Thomson, J. 1864. Systema cerambycidarum ou exposé de tous les genres compris dans la famille des cérambycides et familles limitrophes. Mémoires de la Société Royale des Sciences de Liège, 19:1-540.

1877. Typi cerambycidarum Musei Thomsoniani. Paris, Deyrolle. $21 \mathrm{p}$.

Wendt, H. 1984. Die Cerambyciden-Typen (Coleoptera: Phytophaga) des Zoologischen Museums Berlin. Teil I : Unterfamilie Prioninae. Mitteilungen aus dem Zoologischen Museum in Berlin, 2:327-342.

Recebido em agosto de 2004. Aceito em janeiro de 2005. ISSN 0073-4721

Artigo disponível em: www.scielo.br/isz 